\title{
A Sharp Improvement of a Theorem of Bauer and Schmeichel
}

\author{
Zhora G. Nikoghosyan \\ Institute for Informatics and Automation Problems of NAS RA \\ e-mail: zhora@ipia.sci.am
}

\begin{abstract}
Let $G$ be a graph on $n$ vertices with minimum degree $\delta$. The earliest nontrivial lower bound for the circumference $c$ (the length of a longest cycle in $G$ ) was established in 1952 due to Dirac in terms of $n$ and $\delta$ : (i) if $G$ is a 2-connected graph, then $c \geq \min \{n, 2 \delta\}$. The bound in Theorem $(i)$ is sharp. In 1986, Bauer and Schmeichel gave a version of this classical result for 1-tough graphs: (ii) if $G$ is a 1-tough graph, then $c \geq \min \{n, 2 \delta+2\}$. In this paper we present an improvement of $(i i)$, which is sharp for each $n$ : (iii) if $G$ is a 1-tough graph, then $c \geq \min \{n, 2 \delta+2\}$ when $n \equiv 1(\bmod 3)$; $c \geq \min \{n, 2 \delta+3\}$ when $n \equiv 2(\bmod 3)$ or $n \equiv 1(\bmod 4) ;$ and $c \geq \min \{n, 2 \delta+4\}$ otherwise.
\end{abstract}

Keywords: Hamilton cycle; circumference; minimum degree; 1-tough graphs.

\section{Introduction}

Throughout this article we consider only finite undirected graphs without loops or multiple edges. The set of vertices of a graph $G$ is denoted by $V(G)$ and the set of edges by $E(G)$. We use $n, \delta$ and $c$ to denote the order of $G$, the minimum degree and the circumference the length of a longest cycle in $G$, respectively. A good reference for any undefined terms is $[2]$.

The earliest nontrivial lower bound for the circumference was established in 1952 due to Dirac [4] in terms of $n$ and $\delta$ :

Theorem A [4]: If $G$ is a 2-connected graph, then $c \geq \min \{n, 2 \delta\}$.

The bound $2 \delta$ in Theorem A is sharp.

In 1973, Chvátal [3] introduced the concept of toughness. Since then a lot of research has been done towards finding the exact analogs of classical Hamiltonian results under additional 1-tough condition instead of 2-connectivity - an alternative and stronger necessary condition for a graph to be Hamiltonian.

The analog of the classical Theorem A for 1-tough graphs was established by Bauer and Schmeichel ([1], 1986). 
Theorem B [1]: If $G$ is a 1-tough graph, then $c \geq \min \{n, 2 \delta+2\}$.

The bound $2 \delta+2$ in Theorem B is shown [1] to be sharp by constructing graphs of order $n \equiv 1(\bmod 3)$ with $c=2 \delta+2$.

In this paper we show that the bound $2 \delta+2$ in Theorem $\mathrm{B}$ is sharp if and only if $n \equiv 1(\bmod 3)$. Furthermore, we present a sharp refinement of Theorem $\mathrm{B}$, which is sharp for each $n$.

Theorem 1: Every 1-tough graph is either Hamiltonian, or

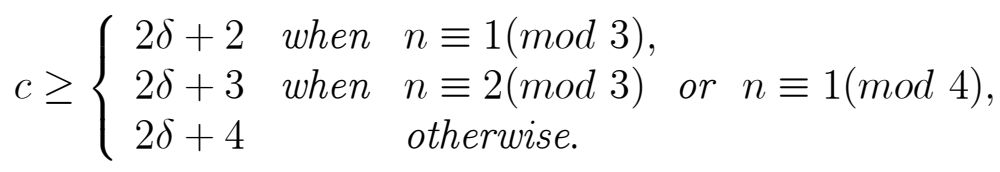

To see that Theorem 1 is sharp for each $n$, let $H_{1}, H_{2}, \ldots, H_{h}$ be disjoint complete graphs with distinct vertices $x_{i}, y_{i} \in V\left(H_{i}\right)(i=1,2, \ldots, h)$. Form a new graph $H\left(t_{1}, t_{2}, \ldots, t_{h}\right)$ by identifying the vertices $x_{1}, x_{2}, \ldots, x_{h}$ and adding all possible edges between $y_{1}, y_{2}, \ldots, y_{h}$, where $t_{i}=\left|V\left(H_{i}\right)\right|(i=1,2, \ldots, h)$. The graph $H(\delta+1, \delta+1, \delta+1)$ shows that the bound $2 \delta+2$ in Theorem 1 cannot be replaced by $2 \delta+3$ when $n \equiv 1(\bmod 3)$. Next, the graphs $H(\delta+2, \delta+1, \delta+1)$ and $H(\delta+1, \delta+1, \delta+1, \delta+1)$ show that the bound $2 \delta+3$ cannot be replaced by $2 \delta+4$ when $n \equiv 2(\bmod 3)$ or $n \equiv 1(\bmod 4)$. Finally, the graph $H(\delta+2, \delta+2, \delta+1)$ shows that the bound $2 \delta+4$ cannot be replaced by $2 \delta+5$.

\section{Notations and Preliminaries}

Let $G$ be a graph. For $S$ a subset of $V(G)$, we denote by $G \backslash S$ the maximum subgraph of $G$ with vertex set $V(G) \backslash S$. We write $\langle S\rangle$ for the subgraph of $G$ induced by $S$. For a subgraph $H$ of $G$ we use $G \backslash H$ short for $G \backslash V(H)$. The neighborhood and the degree of a vertex $x \in V(G)$ will be denoted by $N(x)$ and $d(x)$, respectively. Furthermore, for a subgraph $H$ of $G$ and $x \in V(G)$, we define $N_{H}(x)=N(x) \cap V(H)$ and $d_{H}(x)=\left|N_{H}(x)\right|$. Let $s(G)$ denote the number of components of a graph $G$. A graph $G$ is 1-tough if $|S| \geq s(G \backslash S)$ for every subset $S$ of the vertex set $V(G)$ with $s(G \backslash S)>1$. A graph $G$ on $n$ vertices is Hamiltonian if $G$ contains a Hamilton cycle, i.e., a cycle of length $n$.

Paths and cycles in a graph $G$ are considered as subgraphs of $G$. If $Q$ is a path or a cycle, then the length of $Q$, denoted by $|Q|$, is $|E(Q)|$. We write $Q$ with a given orientation by $\vec{Q}$. For $x, y \in V(Q)$, we denote by $x \vec{Q} y$ the subpath of $Q$ in the chosen direction from $x$ to $y$. For $x \in V(C)$, we denote the $h$-th successor and the $h$-th predecessor of $x$ on $\vec{C}$ by $x^{+h}$ and $x^{-h}$, respectively. We abbreviate $x^{+1}$ and $x^{-1}$ by $x^{+}$and $x^{-}$, respectively. For each $X \subset V(C)$, we define $X^{+}=\left\{x^{+} \mid x \in X\right\}$ and $X^{-}=\left\{x^{-} \mid x \in X\right\}$.

Special definitions. Let $G$ be a graph, $C$ a longest cycle in $G$ and $P=x \vec{P} y$ a longest path in $G \backslash C$ of length $\bar{p} \geq 0$. Let $\xi_{1}, \xi_{2}, \ldots, \xi_{s}$ be the elements of $N_{C}(x) \cup N_{C}(y)$ occurring on $\vec{C}$ in a consecutive order. Set

$$
I_{i}=\xi_{i} \vec{C} \xi_{i+1}, I_{i}^{*}=\xi_{i}^{+} \vec{C} \xi_{i+1}^{-} \quad(i=1,2, \ldots, s),
$$

where $\xi_{s+1}=\xi_{1}$. 
(1) The segments $I_{1}, I_{2}, \ldots, I_{s}$ are called elementary segments on $C$ induced by $N_{C}(x) \cup$ $N_{C}(y)$.

(2) We call a path $L=z \vec{L} w$ an intermediate path between two distinct elementary segments $I_{a}$ and $I_{b}$, if

$$
z \in V\left(I_{a}^{*}\right), w \in V\left(I_{b}^{*}\right), V(L) \cap V(C \cup P)=\{z, w\}
$$

(3) Define $\Upsilon\left(I_{i_{1}}, I_{i_{2}}, \ldots, I_{i_{t}}\right)$ to be the set of all intermediate paths between elementary segments $I_{i_{1}}, I_{i_{2}}, \ldots, I_{i_{t}}$.

(4) If $\Upsilon\left(I_{1}, \ldots, I_{s}\right) \subseteq E$, then the maximum number of intermediate independent edges (not having a common vertex) in $\Upsilon\left(I_{1}, \ldots, I_{s}\right)$ will be denoted by $\mu(\Upsilon)$.

(5) We say that two intermediate independent edges $w_{1} w_{2}, w_{3} w_{4}$ have a crossing, if either $w_{1}, w_{3}, w_{2}, w_{4}$ or $w_{1}, w_{4}, w_{2}, w_{3}$ occur on $\vec{C}$ in a consecutive order.

Lemma 1: Let $G$ be a graph, $C$ a longest cycle in $G$ and $P=x \vec{P}$ y a longest path in $G \backslash C$ of length $\bar{p} \geq 1$. If $\left|N_{C}(x)\right| \geq 2,\left|N_{C}(y)\right| \geq 2$ and $N_{C}(x) \neq N_{C}(y)$, then

$$
c \geq \begin{cases}3 \delta+\max \left\{\sigma_{1}, \sigma_{2}\right\}-1 \geq 3 \delta & \text { if } \quad \bar{p}=1 \\ 4 \delta-2 \bar{p} & \text { if } \quad \bar{p} \geq 2\end{cases}
$$

where $\sigma_{1}=\left|N_{C}(x) \backslash N_{C}(y)\right|$ and $\sigma_{2}=\left|N_{C}(y) \backslash N_{C}(x)\right|$.

Lemma 2: Let $G$ be a graph, $C$ a longest cycle in $G$ and $P=x \vec{P}$ y a longest path in $G \backslash C$ of length $\bar{p} \geq 0$. Let $N_{C}(x)=N_{C}(y),\left|N_{C}(x)\right| \geq 2$ and $f, g \in\{1, \ldots, s\}$.

(a1) If $L \in \Upsilon\left(I_{f}, I_{g}\right)$, then

$$
\left|I_{f}\right|+\left|I_{g}\right| \geq 2 \bar{p}+2|L|+4
$$

(a2) If $\Upsilon\left(I_{f}, I_{g}\right) \subseteq E(G)$ and $\left|\Upsilon\left(I_{f}, I_{g}\right)\right|=\varepsilon$ for some $\varepsilon \in\{1,2,3\}$, then

$$
\left|I_{f}\right|+\left|I_{g}\right| \geq 2 \bar{p}+\varepsilon+5
$$

(a3) If $\Upsilon\left(I_{f}, I_{g}\right) \subseteq E(G)$ and $\Upsilon\left(I_{f}, I_{g}\right)$ contains two independent intermediate edges, then

$$
\left|I_{f}\right|+\left|I_{g}\right| \geq 2 \bar{p}+8
$$

The following result is due to Voss [5].

Lemma 3 [5]: Let $G$ be a Hamiltonian graph, $\left\{v_{1}, v_{2}, \ldots, v_{t}\right\} \subseteq V(G)$ and $d\left(v_{i}\right) \geq t$ $(i=1,2, \ldots, t)$. Then each pair $x, y$ of vertices of $G$ is connected in $G$ by a path of length at least $t$. 


\section{Proofs}

Proof of Lemma 1. Put

$$
A_{1}=N_{C}(x) \backslash N_{C}(y), A_{2}=N_{C}(y) \backslash N_{C}(x), M=N_{C}(x) \cap N_{C}(y) .
$$

By the hypothesis, $N_{C}(x) \neq N_{C}(y)$, implying that

$$
\max \left\{\left|A_{1}\right|,\left|A_{2}\right|\right\} \geq 1 \text {. }
$$

Let $\xi_{1}, \xi_{2}, \ldots, \xi_{s}$ be the elements of $N_{C}(x) \cup N_{C}(y)$ occurring on $\vec{C}$ in a consecutive order. Put $I_{i}=\xi_{i} \vec{C} \xi_{i+1}(i=1,2, \ldots, s)$, where $\xi_{s+1}=\xi_{1}$. Clearly, $s=\left|A_{1}\right|+\left|A_{2}\right|+|M|$. Since $C$ is extreme, we have $\left|I_{i}\right| \geq 2(i=1,2, \ldots, s)$. Next, if $\left\{\xi_{i}, \xi_{i+1}\right\} \cap M \neq \emptyset$ for some $i \in\{1,2, \ldots, s\}$, then $\left|I_{i}\right| \geq \bar{p}+2$. Further, if either $\xi_{i} \in A_{1}, \xi_{i+1} \in A_{2}$ or $\xi_{i} \in A_{2}, \xi_{i+1} \in A_{1}$, then again $\left|I_{i}\right| \geq \bar{p}+2$.

Case 1. $\bar{p}=1$.

Case 1.1. $\left|A_{i}\right| \geq 1(i=1,2)$.

It follows that among $I_{1}, I_{2}, \ldots, I_{s}$ there are $|M|+2$ segments of length at least $\bar{p}+2$. Observing also that each of the remaining $s-(|M|+2)$ segments has a length at least 2 , we have

$$
\begin{gathered}
c \geq(\bar{p}+2)(|M|+2)+2(s-|M|-2) \\
=3(|M|+2)+2\left(\left|A_{1}\right|+\left|A_{2}\right|-2\right)=2\left|A_{1}\right|+2\left|A_{2}\right|+3|M|+2 .
\end{gathered}
$$

Since $\left|A_{1}\right|=d(x)-|M|-1$ and $\left|A_{2}\right|=d(y)-|M|-1$, we have

$$
c \geq 2 d(x)+2 d(y)-|M|-2 \geq 3 \delta+d(x)-|M|-2 .
$$

Recalling that $d(x)=|M|+\left|A_{1}\right|+1$, we get

$$
c \geq 3 \delta+\left|A_{1}\right|-1=3 \delta+\sigma_{1}-1 .
$$

Analogously, $c \geq 3 \delta+\sigma_{2}-1$. So,

$$
c \geq 3 \delta+\max \left\{\sigma_{1}, \sigma_{2}\right\}-1 \geq 3 \delta .
$$

Case 1.2. Either $\left|A_{1}\right| \geq 1,\left|A_{2}\right|=0$ or $\left|A_{1}\right|=0,\left|A_{2}\right| \geq 1$.

Assume w.l.o.g. that $\left|A_{1}\right| \geq 1$ and $\left|A_{2}\right|=0$, i.e. $\left|N_{C}(y)\right|=|M| \geq 2$ and $s=\left|A_{1}\right|+|M|$. Hence, among $I_{1}, I_{2}, \ldots, I_{s}$ there are $|M|+1$ segments of length at least $\bar{p}+2=3$. Taking into account that $|M|+1=d(y)$ and each of the remaining $s-(|M|+1)$ segments has a length at least 2 , we get

$$
\begin{gathered}
c \geq 3(|M|+1)+2(s-|M|-1)=3 d(y)+2\left(\left|A_{1}\right|-1\right) \\
\geq 3 \delta+\left|A_{1}\right|-1=3 \delta+\max \left\{\sigma_{1}, \sigma_{2}\right\}-1 \geq 3 \delta .
\end{gathered}
$$

Case 2. $\bar{p} \geq 2$.

Case 2.1. $\left|A_{i}\right| \geq 1(i=1,2)$.

It follows that among $I_{1}, I_{2}, \ldots, I_{s}$ there are $|M|+2$ segments of length at least $\bar{p}+2$. Further, since each of the remaining $s-(|M|+2)$ segments has a length at least 2 , we get

$$
c \geq(\bar{p}+2)(|M|+2)+2(s-|M|-2)
$$




$$
\begin{gathered}
=(\bar{p}-2)|M|+(2 \bar{p}+4|M|+4)+2\left(\left|A_{1}\right|+\left|A_{2}\right|-2\right) \\
\geq 2\left|A_{1}\right|+2\left|A_{2}\right|+4|M|+2 \bar{p} .
\end{gathered}
$$

Observing also that

$$
\left|A_{1}\right|+|M|+\bar{p} \geq d(x), \quad\left|A_{2}\right|+|M|+\bar{p} \geq d(y),
$$

we have

$$
\begin{gathered}
2\left|A_{1}\right|+2\left|A_{2}\right|+4|M|+2 \bar{p} \\
\geq 2 d(x)+2 d(y)-2 \bar{p} \geq 4 \delta-2 \bar{p},
\end{gathered}
$$

implying that $c \geq 4 \delta-2 \bar{p}$.

Case 2.2. Either $\left|A_{1}\right| \geq 1,\left|A_{2}\right|=0$ or $\left|A_{1}\right|=0,\left|A_{2}\right| \geq 1$.

Assume w.l.o.g. that $\left|A_{1}\right| \geq 1$ and $\left|A_{2}\right|=0$, that is $\left|N_{C}(y)\right|=|M| \geq 2$ and $s=\left|A_{1}\right|+|M|$. It follows that among $I_{1}, I_{2}, \ldots, I_{s}$ there are $|M|+1$ segments of length at least $\bar{p}+2$. Observing also that $|M|+\bar{p} \geq d(y) \geq \delta$, i.e., $2 \bar{p}+4|M| \geq 4 \delta-2 \bar{p}$, we get

$$
\begin{gathered}
c \geq(\bar{p}+2)(|M|+1) \geq(\bar{p}-2)(|M|-1)+2 \bar{p}+4|M| \\
\geq 2 \bar{p}+4|M| \geq 4 \delta-2 \bar{p} .
\end{gathered}
$$

Proof of Lemma 2. Let $\xi_{1}, \xi_{2}, \ldots, \xi_{s}$ be the elements of $N_{C}(x)$ occurring on $\vec{C}$ in a consecutive order. Put $I_{i}=\xi_{i} \vec{C} \xi_{i+1}(i=1,2, \ldots, s)$, where $\xi_{s+1}=\xi_{1}$. To prove $(a 1)$, let $L \in \Upsilon\left(I_{f}, I_{g}\right)$. Further, let $L=z \vec{L} w$ with $z \in V\left(I_{f}^{*}\right)$ and $w \in V\left(I_{g}^{*}\right)$. Put

$$
\begin{gathered}
\left|\xi_{f} \vec{C} z\right|=d_{1},\left|z \vec{C} \xi_{f+1}\right|=d_{2},\left|\xi_{g} \vec{C} w\right|=d_{3},\left|w \vec{C} \xi_{g+1}\right|=d_{4} \\
C^{\prime}=\xi_{f} x \vec{P} y \xi_{g} \overleftarrow{C} z \vec{L} w \vec{C} \xi_{f} .
\end{gathered}
$$

Clearly,

$$
\left|C^{\prime}\right|=|C|-d_{1}-d_{3}+|L|+|P|+2 .
$$

Since $C$ is extreme, we have $|C| \geq\left|C^{\prime}\right|$, implying that $d_{1}+d_{3} \geq \bar{p}+|L|+2$. By a symmetric argument, $d_{2}+d_{4} \geq \bar{p}+|L|+2$. Hence

$$
\left|I_{f}\right|+\left|I_{g}\right|=\sum_{i=1}^{4} d_{i} \geq 2 \bar{p}+2|L|+4 .
$$

The proof of $(a 1)$ is complete. To prove $(a 2)$ and $(a 3)$, let $\Upsilon\left(I_{f}, I_{g}\right) \subseteq E(G)$ and $\left|\Upsilon\left(I_{f}, I_{g}\right)\right|=\varepsilon$ for some $\varepsilon \in\{1,2,3\}$.

Case 1. $\varepsilon=1$.

Let $L \in \Upsilon\left(I_{f}, I_{g}\right)$, where $|L|=1$. By (a1),

$$
\left|I_{f}\right|+\left|I_{g}\right| \geq 2 \bar{p}+2|L|+4=2 \bar{p}+6 .
$$

Case 2. $\varepsilon=2$. 
It follows that $\Upsilon\left(I_{f}, I_{g}\right)$ consists of two edges $e_{1}, e_{2}$. Put $e_{1}=z_{1} w_{1}$ and $e_{2}=z_{2} w_{2}$, where $\left\{z_{1}, z_{2}\right\} \subseteq V\left(I_{f}^{*}\right)$ and $\left\{w_{1}, w_{2}\right\} \subseteq V\left(I_{g}^{*}\right)$.

Case 2.1. $z_{1} \neq z_{2}$ and $w_{1} \neq w_{2}$.

Assume w.l.o.g. that $z_{1}$ and $z_{2}$ occur in this order on $I_{f}$.

Case 2.1.1. $w_{2}$ and $w_{1}$ occur in this order on $I_{g}$.

Put

$$
\begin{gathered}
\left|\xi_{f} \vec{C} z_{1}\right|=d_{1},\left|z_{1} \vec{C} z_{2}\right|=d_{2},\left|z_{2} \vec{C} \xi_{f+1}\right|=d_{3} \\
\left|\xi_{g} \vec{C} w_{2}\right|=d_{4},\left|w_{2} \vec{C} w_{1}\right|=d_{5},\left|w_{1} \vec{C} \xi_{g+1}\right|=d_{6} \\
C^{\prime}=\xi_{f} \vec{C} z_{1} w_{1} \overleftarrow{C} w_{2} z_{2} \vec{C} \xi_{g} x \vec{P} y \xi_{g+1} \vec{C} \xi_{f}
\end{gathered}
$$

Clearly,

$$
\begin{gathered}
\left|C^{\prime}\right|=|C|-d_{2}-d_{4}-d_{6}+\left|\left\{e_{1}\right\}\right|+\left|\left\{e_{2}\right\}\right|+|P|+2 \\
=|C|-d_{2}-d_{4}-d_{6}+\bar{p}+4 .
\end{gathered}
$$

Since $C$ is extreme, we have $|C| \geq\left|C^{\prime}\right|$, implying that $d_{2}+d_{4}+d_{6} \geq \bar{p}+4$. By a symmetric argument, $d_{1}+d_{3}+d_{5} \geq \bar{p}+4$. Hence

$$
\left|I_{f}\right|+\left|I_{g}\right|=\sum_{i=1}^{6} d_{i} \geq 2 \bar{p}+8
$$

Case 2.1.2. $w_{1}$ and $w_{2}$ occur in this order on $I_{g}$.

Putting

$$
C^{\prime}=\xi_{f} \vec{C} z_{1} w_{1} \vec{C} w_{2} z_{2} \vec{C} \xi_{g} x \vec{P} y \xi_{g+1} \vec{C} \xi_{f},
$$

we can argue as in Case 2.1.1.

Case 2.2. Either $z_{1}=z_{2}, w_{1} \neq w_{2}$ or $z_{1} \neq z_{2}, w_{1}=w_{2}$.

Assume w.l.o.g. that $z_{1} \neq z_{2}, w_{1}=w_{2}$ and $z_{1}, z_{2}$ occur in this order on $I_{f}$. Put

$$
\begin{gathered}
\left|\xi_{f} \vec{C} z_{1}\right|=d_{1},\left|z_{1} \vec{C} z_{2}\right|=d_{2},\left|z_{2} \vec{C} \xi_{f+1}\right|=d_{3}, \\
\left|\xi_{g} \vec{C} w_{1}\right|=d_{4},\left|w_{1} \vec{C} \xi_{g+1}\right|=d_{5}, \\
C^{\prime}=\xi_{f} x \vec{P} y \xi_{g} \overleftarrow{C} z_{1} w_{1} \vec{C} \xi_{f}, \\
C^{\prime \prime}=\xi_{f} \vec{C} z_{2} w_{1} \overleftarrow{C} \xi_{f+1} x \vec{P} y \xi_{g+1} \vec{C} \xi_{f} .
\end{gathered}
$$

Clearly,

$$
\begin{aligned}
& \left|C^{\prime}\right|=|C|-d_{1}-d_{4}+\left|\left\{e_{1}\right\}\right|+|P|+2=|C|-d_{1}-d_{4}+\bar{p}+3, \\
& \left|C^{\prime \prime}\right|=|C|-d_{3}-d_{5}+\left|\left\{e_{2}\right\}\right|+|P|+2=|C|-d_{3}-d_{5}+\bar{p}+3 .
\end{aligned}
$$

Since $C$ is extreme, $|C| \geq\left|C^{\prime}\right|$ and $|C| \geq\left|C^{\prime \prime}\right|$, implying that

$$
d_{1}+d_{4} \geq \bar{p}+3, d_{3}+d_{5} \geq \bar{p}+3 .
$$


Hence,

$$
\left|I_{f}\right|+\left|I_{g}\right|=\sum_{i=1}^{5} d_{i} \geq d_{1}+d_{3}+d_{4}+d_{5}+1 \geq 2 \bar{p}+7
$$

Case 3. $\varepsilon=3$.

It follows that $\Upsilon\left(I_{f}, I_{g}\right)$ consists of three edges $e_{1}, e_{2}, e_{3}$. Let $e_{i}=z_{i} w_{i}(i=1,2,3)$, where $\left\{z_{1}, z_{2}, z_{3}\right\} \subseteq V\left(I_{f}^{*}\right)$ and $\left\{w_{1}, w_{2}, w_{3}\right\} \subseteq V\left(I_{g}^{*}\right)$. If there are two independent edges among $e_{1}, e_{2}, e_{3}$, then we can argue as in Case 2.1. Otherwise, we can assume w.l.o.g. that $w_{1}=w_{2}=w_{3}$ and $z_{1}, z_{2}, z_{3}$ occur in this order on $I_{f}$. Put

$$
\begin{gathered}
\left|\xi_{f} \vec{C} z_{1}\right|=d_{1},\left|z_{1} \vec{C} z_{2}\right|=d_{2},\left|z_{2} \vec{C} z_{3}\right|=d_{3}, \\
\left|z_{3} \vec{C} \xi_{f+1}\right|=d_{4},\left|\xi_{g} \vec{C} w_{1}\right|=d_{5},\left|w_{1} \vec{C} \xi_{g+1}\right|=d_{6} \\
C^{\prime}=\xi_{f} x \vec{P} y \xi_{g} \overleftarrow{C} z_{1} w_{1} \vec{C} \xi_{f} \\
C^{\prime \prime}=\xi_{f} \vec{C} z_{3} w_{1} \overleftarrow{C} \xi_{f+1} x \vec{P} y \xi_{g+1} \vec{C} \xi_{f}
\end{gathered}
$$

Clearly,

$$
\begin{aligned}
& \left|C^{\prime}\right|=|C|-d_{1}-d_{5}+\left|\left\{e_{1}\right\}\right|+\bar{p}+2, \\
& \left|C^{\prime \prime}\right|=|C|-d_{4}-d_{6}+\left|\left\{e_{3}\right\}\right|+\bar{p}+2 .
\end{aligned}
$$

Since $C$ is extreme, we have $|C| \geq\left|C^{\prime}\right|$ and $|C| \geq\left|C^{\prime \prime}\right|$, implying that

$$
d_{1}+d_{5} \geq \bar{p}+3, d_{4}+d_{6} \geq \bar{p}+3
$$

Hence,

$$
\left|I_{f}\right|+\left|I_{g}\right|=\sum_{i=1}^{6} d_{i} \geq d_{1}+d_{4}+d_{5}+d_{6}+2 \geq 2 \bar{p}+8
$$

Proof of Theorem 1. Let $G$ be a 1-tough graph. If $c \geq 2 \delta+4$, then we are done. Hence, we can assume that

$$
c \leq 2 \delta+3 \text {. }
$$

Let $C$ be a longest cycle in $G$ and $P=x_{1} \vec{P} x_{2}$ a longest path in $G \backslash C$. Put $|P|=$ $|V(P)|-1=\bar{p}$. If $|V(P)|=0$, then $C$ is a Hamilton cycle and we are done. Let $|V(P)| \geq 1$, that is $\bar{p} \geq 0$. Put $X=N_{C}\left(x_{1}\right) \cup N_{C}\left(x_{2}\right)$ and let $\xi_{1}, \ldots, \xi_{s}$ be the elements of $X$ occurring on $C$ in a consecutive order. Put

$$
I_{i}=\xi_{i} \vec{C} \xi_{i+1}, I_{i}^{*}=\xi_{i}^{+} \vec{C} \xi_{i+1}^{-}(i=1, \ldots, s),
$$

where $\xi_{s+1}=\xi_{1}$. Since $G$ is a 1 -tough graph, we have $\delta \geq 2$.

Case 1. $\bar{p} \leq \delta-2$.

It follows that $s \geq\left|N_{C}\left(x_{i}\right)\right| \geq \delta-\bar{p} \geq 2(i=1,2)$. Assume first that $N_{C}\left(x_{1}\right) \neq N_{C}\left(x_{2}\right)$, implying that $\bar{p} \geq 1$. If $\bar{p} \geq 2$, then by Lemma $1, c \geq 4 \delta-2 \bar{p} \geq 2 \delta+4$, contradicting (1). Hence $\bar{p}=1$, which yields $\delta \geq \bar{p}+2=3$. By Lemma $1, c \geq 3 \delta \geq 9$. If $\delta \geq 4$, then $c \geq 3 \delta \geq 2 \delta+4$, contradicting (1). Let $\delta=3$. Next, we can suppose that $c=9$, since otherwise $c \geq 10=3 \delta+1=2 \delta+4$, contradicting (1). Further, we can suppose that $s \geq 3$, since $N_{C}\left(x_{1}\right)=N_{C}\left(x_{2}\right)$ when $s=2$, contradicting the hypothesis. Finally, we can suppose 
that $s=3$, since clearly $c \geq 10$ when $s \geq 4$, a contradiction. Thus, $\left|I_{1}\right|=\left|I_{2}\right|=\left|I_{3}\right|=3$ and it is not hard to see that $G \backslash\left\{\xi_{1}, \xi_{2}, \xi_{3}\right\}$ has at least four components, contradicting $\tau \geq 1$.

Now assume that $N_{C}\left(x_{1}\right)=N_{C}\left(x_{2}\right)$. Since $C$ is extreme, we have

$$
\left|I_{i}\right| \geq\left|\xi_{i} x_{1} \vec{P} x_{2} \xi_{i+1}\right| \geq \bar{p}+2 \quad(i=1, \ldots, s) .
$$

Case 1.1. $s \geq \delta-\bar{p}+1$.

Clearly,

$$
\begin{gathered}
c=\sum_{i=1}^{s}\left|I_{i}\right| \geq s(\bar{p}+2) \\
\geq(\delta-\bar{p}+1)(\bar{p}+2)=(\delta-\bar{p}-2) \bar{p}+2 \delta+\bar{p}+2 .
\end{gathered}
$$

If $\bar{p} \geq 2$, then by (2), $c \geq 2 \delta+4$, contradicting (1). Let $\bar{p} \leq 1$.

Case 1.1.1. $\bar{p}=0$.

If $\Upsilon\left(I_{1}, \ldots, I_{s}\right)=\emptyset$, then $G \backslash\left\{\xi_{1}, \ldots, \xi_{s}\right\}$ has at least $s+1$ components, contradicting the fact that $\tau \geq 1$. Otherwise $\Upsilon\left(I_{a}, I_{b}\right) \neq \emptyset$ for some distinct $a, b \in\{1, \ldots, s\}$. Let $L \in \Upsilon\left(I_{a}, I_{b}\right)$. By Lemma 2(a1),

$$
\left|I_{a}\right|+\left|I_{b}\right| \geq 2 \bar{p}+2|L|+4 \geq 6 .
$$

Recalling also that $s \geq \delta-\bar{p}+1=\delta+1$, we get

$$
c=\sum_{i=1}^{s}\left|I_{i}\right| \geq\left|I_{a}\right|+\left|I_{b}\right|+2(s-2)=2 s+2 \geq 2 \delta+4
$$

contradicting (1).

Case 1.1.2. $\bar{p}=1$.

By (2), $c \geq 3 \delta$. We can suppose that $\delta \leq 3$, since $c \geq 3 \delta \geq 2 \delta+4$ when $\delta \geq 4$, contradicting (1). On the other hand, by the hypothesis, $\delta \geq \bar{p}+2=3$, implying that $\delta=3$. By the hypothesis, $s \geq \delta-\bar{p}+1=3$. Next, we can suppose that $s=3$, since $c \geq s(\bar{p}+2) \geq 12=2 \delta+6$ when $s \geq 4$, contradicting (1). Further, if $\Upsilon\left(I_{1}, I_{2}, I_{3}\right)=\emptyset$, then $G \backslash\left\{\xi_{1}, \xi_{2}, \xi_{3}\right\}$ has at least four components, contradicting $\tau \geq 1$. Otherwise $\Upsilon\left(I_{a}, I_{b}\right) \neq \emptyset$ for some distinct $a, b \in\{1,2,3\}$, say $a=1$ and $b=2$. Let $L \in \Upsilon\left(I_{1}, I_{2}\right)$. By Lemma 2(a1),

$$
\left|I_{1}\right|+\left|I_{2}\right| \geq 2 \bar{p}+2|L|+4=8
$$

which yields $c \geq\left|I_{1}\right|+\left|I_{2}\right|+\left|I_{3}\right| \geq 11=2 \delta+5$, contradicting (1).

Case 1.2. $s=\delta-\bar{p}$.

It follows that $x_{1} x_{2} \in E$. Then $x_{1} x_{2} \overleftarrow{P} x_{1}^{+}$is another longest path in $G \backslash C$. We can suppose that $N_{C}\left(x_{1}\right)=N_{C}\left(x_{1}^{+}\right)$, since otherwise we can argue as in Case 1 . By the same reason,

$$
N_{C}\left(x_{1}\right)=N_{C}\left(x_{1}^{+}\right)=N_{C}\left(x_{1}^{+2}\right)=\ldots=N_{C}\left(x_{2}\right) .
$$

Since $C$ is extreme, we have $\left|I_{i}\right| \geq\left|\xi_{i} x_{1} \vec{P} x_{2} \xi_{i+1}\right|=\bar{p}+2(i=1, \ldots, s)$. If $\Upsilon\left(I_{1}, \ldots, I_{s}\right)=\emptyset$, then $G \backslash\left\{\xi_{1}, \ldots, \xi_{s}\right\}$ has at least $s+1$ components, contradicting $\tau \geq 1$. Otherwise $\Upsilon\left(I_{a}, I_{b}\right) \neq$ 
$\emptyset$ for some distinct $a, b \in\{1, \ldots, s\}$. Let $L \in \Upsilon\left(I_{a}, I_{b}\right)$ with $L=z_{1} \vec{L} z_{2}$, where $z_{1} \in V\left(I_{a}^{*}\right)$ and $z_{2} \in V\left(I_{b}^{*}\right)$. By Lemma 2(a1), $\left|I_{a}\right|+\left|I_{b}\right| \geq 2 \bar{p}+6$. Hence

$$
\begin{gathered}
c=\sum_{i=1}^{s}\left|I_{i}\right| \geq\left|I_{a}\right|+\left|I_{b}\right|+(s-2)(\bar{p}+2) \geq s(\bar{p}+2)+2 \\
=(\delta-\bar{p})(\bar{p}+2)+2=2 \delta+2+\bar{p}(\delta-\bar{p}-2) .
\end{gathered}
$$

Claim 1. (a1) $2 \bar{p}+6 \leq\left|I_{a}\right|+\left|I_{b}\right| \leq 2 \bar{p}+7$ and $\left|I_{i}\right| \leq \bar{p}+5(i=1, \ldots, s)$.

(a2) If $\left|I_{a}\right|+\left|I_{b}\right|=2 \bar{p}+7$, then $\left|I_{i}\right|=\bar{p}+2$ for each $i \in\{1, \ldots, s\} \backslash\{a, b\}$.

(a3) If $\left|I_{a}\right|+\left|I_{b}\right|=2 \bar{p}+6$, then $\left|I_{f}\right| \leq \bar{p}+3$ for some $f \in\{1, \ldots, s\} \backslash\{a, b\}$ and $\left|I_{i}\right|=\bar{p}+2$ for each $i \in\{1, \ldots, s\} \backslash\{a, b, f\}$.

(a4) If $\left|I_{f}\right|=\bar{p}+5$ for some $f \in\{a, b\}$, then $\left|I_{i}\right|=\bar{p}+2$ for each $i \in\{1, \ldots, s\} \backslash\{f\}$.

(a5) For each distinct $f, g, h \in\{1, \ldots, s\},\left|I_{f}\right|+\left|I_{g}\right|+\left|I_{h}\right| \leq 3 \bar{p}+9$.

(a6) $\Upsilon\left(I_{1}, \ldots, I_{s}\right) \subseteq E$.

Proof. If $\left|I_{f}\right| \geq \bar{p}+6$ for some $f \in\{1, \ldots, s\}$, then

$$
\begin{gathered}
c=\sum_{i=1}^{s}\left|I_{i}\right| \geq\left|I_{f}\right|+(s-1)(\bar{p}+2) \geq s(\bar{p}+2)+4 \\
=2 \delta+4+\bar{p}(\delta-\bar{p}-2) \geq 2 \delta+4
\end{gathered}
$$

contradicting (1). Next, if $\left|I_{a}\right|+\left|I_{b}\right| \geq 2 \bar{p}+8$, then

$$
c \geq\left|I_{a}\right|+\left|I_{b}\right|+(s-2)(\bar{p}+2) \geq s(\bar{p}+2)+4 \geq 2 \delta+4,
$$

again contradicting (1). Hence $(a 1)$ holds. Statements $(a 2)-(a 4)$ can be proved by a similar way. To prove (a5), assume the contrary, that is $\left|I_{f}\right|+\left|I_{g}\right|+\left|I_{h}\right| \geq 3 \bar{p}+10$ for some distinct $f, g, h \in\{1, \ldots, s\}$. Then

$$
\begin{gathered}
c=\sum_{i=1}^{s}\left|I_{i}\right| \geq\left|I_{f}\right|+\left|I_{g}\right|+\left|I_{h}\right|+(s-3)(\bar{p}+2) \\
\geq 3(\bar{p}+2)+4+(s-3)(\bar{p}+2)=2 \delta+4+\bar{p}(s-2) \geq 2 \delta+4,
\end{gathered}
$$

contradicting (1). Statement (a6) follows from Lemma 2(a1) and Claim 1(a1). Claim 1 is proved.

Claim 2. $\bar{p}+3 \leq d_{1} \leq \bar{p}+4$ and $\bar{p}+3 \leq d_{2} \leq \bar{p}+4$, where

$$
d_{1}=\left|\xi_{a} \vec{C} z_{1}\right|+\left|\xi_{b} \vec{C} z_{2}\right|, \quad d_{2}=\left|z_{1} \vec{C} \xi_{a+1}\right|+\left|z_{2} \vec{C} \xi_{b+1}\right| .
$$

Proof. Put

$$
Q=\xi_{a} x_{1} \vec{P} x_{2} \xi_{b} \overleftarrow{C} z_{1} z_{2} \vec{C} \xi_{a}
$$

Clearly, $|Q|=|C|-d_{1}+\bar{p}+3$. Since $C$ is extreme, we have $|C| \geq|Q|$, implying that $d_{1} \geq \bar{p}+3$. By a symmetric argument, $d_{2} \geq \bar{p}+3$. By Claim 1(a1), $\left|I_{a}\right|+\left|I_{b}\right|=d_{1}+d_{2} \leq 2 \bar{p}+7$. If $d_{1} \geq \bar{p}+5$, then $2 \bar{p}+7 \geq d_{1}+d_{2} \geq \bar{p}+5+d_{2}$, implying that $d_{2} \leq \bar{p}+2$, a contradiction. Hence, $d_{1} \leq \bar{p}+4$. By a symmetric argument, $d_{2} \leq \bar{p}+4$. Claim 2 is proved.

Claim 3. If $v_{1} \in V\left(\xi_{a}^{+} \vec{C} z_{1}^{-}\right)$and $v_{2} \in V\left(z_{1}^{+} \vec{C} \xi_{a+1}^{-}\right)$, then $v_{1} v_{2} \notin E$. 
Proof. Assume the contrary, that is $v_{1} v_{2} \in E$. Put

$$
\begin{gathered}
Q=\xi_{a} \vec{C} v_{1} v_{2} \overleftarrow{C} z_{1} z_{2} \overleftarrow{C} \xi_{a+1} x_{1} \vec{P} x_{2} \xi_{b+1} \vec{C} \xi_{a} \\
\left|\xi_{a} \vec{C} v_{1}\right|=d_{1}, \quad\left|v_{1} \vec{C} z_{1}\right|=d_{2}, \quad\left|z_{1} \vec{C} v_{2}\right|=d_{3}, \\
\left|v_{2} \vec{C} \xi_{a+1}\right|=d_{4}, \quad\left|\xi_{b} \vec{C} z_{2}\right|=d_{5}, \quad\left|z_{2} \vec{C} \xi_{b+1}\right|=d_{6}
\end{gathered}
$$

Clearly, $|Q|=|C|-d_{2}-d_{4}-d_{6}+\bar{p}+4$. Since $C$ is extreme, we have $|Q| \leq|C|$, implying that $d_{2}+d_{4}+d_{6} \geq \bar{p}+4$. By a symmetric argument, $d_{1}+d_{3}+d_{5} \geq \bar{p}+4$. By summing, we get

$$
\sum_{i=1}^{6} d_{i}=\left|I_{a}\right|+\left|I_{b}\right| \geq 2 \bar{p}+8
$$

contradicting Claim $1(\mathrm{a} 1)$. Thus, $v_{1} v_{2} \notin E$. Claim 3 is proved.

Claim 4. Let $\xi_{f}, \xi_{g}, \xi_{h}$ occur on $\vec{C}$ in a consecutive order for some $f, g, h \in\{1, \ldots, s\}$ and $w_{1} w_{2} \in E$ for some $w_{1} \in V\left(I_{f}^{*}\right)$ and $w_{2} \in V\left(I_{g}^{*}\right)$. If $N\left(w_{3}\right) \cap\left\{\xi_{f+1}, \xi_{g}\right\} \neq \emptyset$ for some $w_{3} \in V\left(I_{h}^{*}\right)$, then

$$
\left|w_{1} \vec{C} \xi_{f+1}\right|+\left|\xi_{g} \vec{C} w_{2}\right|+\left|\xi_{h} \vec{C} w_{3}\right| \geq \bar{p}+4 .
$$

Further, if $N\left(w_{4}\right) \cap\left\{\xi_{f+1}, \xi_{g}\right\} \neq \emptyset$ for some $w_{4} \in V\left(I_{h-1}^{*}\right)$, then

$$
\left|w_{1} \vec{C} \xi_{f+1}\right|+\left|\xi_{g} \vec{C} w_{2}\right|+\left|w_{4} \vec{C} \xi_{h}\right| \geq \bar{p}+4 .
$$

Proof. Assume first that $w_{3} \xi_{f+1} \in E$. Put

$$
Q=\xi_{f} \vec{C} w_{1} w_{2} \vec{C} \xi_{h} x_{1} \vec{P} x_{2} \xi_{g} \overleftarrow{C} \xi_{f+1} w_{3} \xi_{f}
$$

Clearly,

$$
|Q|=|C|-\left|w_{1} \vec{C} \xi_{f+1}\right|-\left|\xi_{g} \vec{C} w_{2}\right|-\left|\xi_{h} \vec{C} w_{3}\right|+\bar{p}+4 .
$$

Since $|Q| \leq|C|$, the desired result holds immediately. If $w_{4} \xi_{f+1} \in E$, then we can use the following cycle

$$
Q^{\prime}=\xi_{f} \vec{C} w_{1} w_{2} \vec{C} w_{4} \xi_{f+1} \vec{C} \xi_{g} x_{2} \overleftarrow{P} x_{1} \xi_{h} \vec{C} \xi_{f}
$$

instead of $Q$. By a symmetric argument, the desired result holds when either $w_{3} \xi_{g} \in E$ or $w_{4} \xi_{g} \in E$. Claim 4 is proved.

Claim 5. Every two intermediate independent edges $e_{1}, e_{2}$ in $\Upsilon\left(I_{1}, \ldots, I_{s}\right)$ have a crossing with $e_{1}, e_{2} \in \Upsilon\left(I_{f}, I_{g}, I_{h}\right)$ for some distinct $f, g, h \in\{1, \ldots, s\}$.

Proof. Let $e_{1}=w_{1} w_{2}$ and $e_{2}=w_{3} w_{4}$. We distinguish three different cases. First, if $e_{1}, e_{2} \in \Upsilon\left(I_{f}, I_{g}\right)$ for some distinct $f, g$, then by Lemma $2(\mathrm{a} 3),\left|I_{f}\right|+\left|I_{g}\right| \geq 2 \bar{p}+8$, contradicting Claim 1(a1). Next, if $e_{1} \in \Upsilon\left(I_{f}, I_{g}\right)$ and $e_{2} \in \Upsilon\left(I_{h}, I_{r}\right)$ for some distinct $f, g, h, r$, then by Lemma $2\left(\right.$ a1), $\left|I_{f}\right|+\left|I_{g}\right| \geq 2 \bar{p}+6$ and $\left|I_{h}\right|+\left|I_{r}\right| \geq 2 \bar{p}+6$, implying that

$$
\begin{gathered}
c \geq\left|I_{f}\right|+\left|I_{g}\right|+\left|I_{h}\right|+\left|I_{r}\right|+(s-4)(\bar{p}+2)=4 \bar{p}+12+(s-4)(\bar{p}+2) \\
=s(\bar{p}+2)+4=2 \delta+4+\bar{p}(\delta-\bar{p}-2) \geq 2 \delta+4,
\end{gathered}
$$

which again contradicts (1). Finally, let $e_{1} \in \Upsilon\left(I_{f}, I_{g}\right)$ and $e_{2} \in \Upsilon\left(I_{f}, I_{h}\right)$ for some distinct $f, g, h$. Assume w.l.o.g. that $\xi_{f}, \xi_{g}, \xi_{h}$ occur on $\vec{C}$ in a consecutive order and $w_{1}, w_{3} \in V\left(I_{f}^{*}\right)$, 
$w_{2} \in V\left(I_{g}^{*}\right), w_{4} \in V\left(I_{h}^{*}\right)$. We can assume also that $w_{3}$ and $w_{1}$ occur on $I_{f}$ in a consecutive order, since otherwise $e_{1}$ and $e_{2}$ have a crossing and we are done. Put

$$
\begin{gathered}
Q=\xi_{f} \vec{C} w_{3} w_{4} \overleftarrow{C} w_{2} w_{1} \vec{C} \xi_{g} x_{2} \overleftarrow{P} x_{1} \xi_{h+1} \vec{C} \xi_{f} \\
\left|\xi_{f} \vec{C} w_{3}\right|=d_{1},\left|w_{3} \vec{C} w_{1}\right|=d_{2},\left|w_{1} \vec{C} \xi_{f+1}\right|=d_{3} \\
\left|\xi_{g} \vec{C} w_{2}\right|=d_{4},\left|w_{2} \vec{C} \xi_{g+1}\right|=d_{5},\left|\xi_{h} \vec{C} w_{4}\right|=d_{6},\left|w_{4} \vec{C} \xi_{h+1}\right|=d_{7}
\end{gathered}
$$

Clearly, $|Q|=|C|-d_{2}-d_{4}-d_{7}+\bar{p}+4$. Since $C$ is extreme, we have $|Q| \leq|C|$, implying that $d_{2}+d_{4}+d_{7} \geq \bar{p}+4$. On the other hand, by Lemma $2, d_{3}+d_{5} \geq \bar{p}+3$ and $d_{1}+d_{6} \geq \bar{p}+3$. By summing, we get $\sum_{i=1}^{7} d_{i}=\left|I_{f}\right|+\left|I_{g}\right|+\left|I_{h}\right| \geq 3 \bar{p}+10$. Then

$$
|C| \geq\left|I_{f}\right|+\left|I_{g}\right|+\left|I_{h}\right|+(s-3)(\bar{p}+2)=s(\bar{p}+2)+4 \geq 2 \delta+4,
$$

contradicting (1). Claim 5 is proved.

Claim 6. If $\mu(\Upsilon)=1$, then $s \leq 3$ and either $\xi_{a}^{+} \xi_{b+1}^{-} \in E$ with $\xi_{a}=\xi_{b+1}$ or $\xi_{a+1}^{-} \xi_{b}^{+} \in E$ with $\xi_{a+1}=\xi_{b}$. If $\mu(\Upsilon)=1$ and $s=3$, then $\left|I_{1}\right|=\left|I_{2}\right|=\left|I_{3}\right|=\bar{p}+3$.

Proof. Since $\mu(\Upsilon)=1$, either one of the vertices $z_{1}, z_{2}$, say $z_{1}$, is a common vertex for all edges in $\Upsilon\left(I_{1}, \ldots, I_{s}\right)$ or $z_{1} z_{3}, z_{2} z_{3} \in \Upsilon\left(I_{1}, \ldots, I_{s}\right)$ for some $z_{3} \in V\left(I_{f}^{*}\right)$ and $f \in\{1, \ldots, s\} \backslash\{a, b\}$.

Case a1. $z_{1}$ is a common vertex for all edges in $\Upsilon\left(I_{1}, \ldots, I_{s}\right)$.

If $z_{1} \notin\left\{\xi_{a}^{+}, \xi_{a+1}^{-}\right\}$, then by Claim $3, G \backslash\left\{\xi_{1}, \ldots, \xi_{s}, z_{1}\right\}$ has at least $s+2$ components, contradicting $\tau \geq 1$. Let $z_{1} \in\left\{\xi_{a}^{+}, \xi_{a+1}^{-}\right\}$, say $z_{1}=\xi_{a}^{+}$.

Case a1.1. $z_{1} \xi_{b+1}^{-} \notin E$.

It follows that $z_{2} \neq \xi_{b+1}^{-}$. By Claim $2,\left|\xi_{b} \vec{C} z_{2}\right| \geq \bar{p}+2$.

Case a1.1.1. $z_{1} \xi_{b+1}^{-2} \notin E$.

It follows that $\left|I_{b}\right| \geq \bar{p}+5$. By Claim 1(a1), $\left|I_{a}\right|=\bar{p}+2$. Moreover, we have $\left|I_{b}\right|=\bar{p}+5$, $\left|\xi_{b} \vec{C} z_{2}\right|=\bar{p}+2, z_{2}=\xi_{b+1}^{-3}$ and $N\left(z_{1}\right) \cap V\left(I_{b}^{*}\right)=\left\{z_{2}\right\}$. By Claim $1(\mathrm{a} 4),\left|I_{i}\right|=\bar{p}+2$ for each $i \in\{1, \ldots, s\} \backslash\{b\}$. Next, by Lemma $2(\mathrm{a} 1), \Upsilon\left(I_{a}, I_{i}\right)=\emptyset$ for each $i \in\{1, \ldots, s\} \backslash\{a, b\}$. Thus, if $z_{1} y \in \Upsilon\left(I_{1}, \ldots, I_{s}\right)$, then $y=z_{2}$, implying that $\Upsilon\left(I_{1}, \ldots, I_{s}\right)=\left\{z_{1} z_{2}\right\}$. Besides, since $\left|\xi_{b} \vec{C} z_{2}\right|=\bar{p}+2 \geq 2$, we have $z_{2} \notin\left\{\xi_{b}^{+}, \xi_{b+1}^{-}\right\}$. Therefore, by Claim $3, G \backslash\left\{\xi_{1}, \ldots, \xi_{s}, z_{2}\right\}$ has at least $s+2$ components, contradicting $\tau \geq 1$.

Case a1.1.2. $z_{1} \xi_{b+1}^{-2} \in E$.

It follows that $\left|I_{b}\right| \geq \bar{p}+4$. Assume first that $\left|I_{b}\right|=\bar{p}+5$. If $z_{1} \xi_{b+1}^{-3} \notin E$, then clearly $z_{2}=\xi_{b+1}^{-2}$ and we can argue as in Case a1.1.1. Otherwise the following cycle

$$
\xi_{a} x_{1} \vec{P} x_{2} \xi_{a+1} \vec{C} \xi_{b+1}^{-3} z_{1} \xi_{b+1}^{-2} \vec{C} \xi_{a}
$$

is longer than $C$, a contradiction.

Now assume that $\left|I_{b}\right|=\bar{p}+4$, that is $\left|\xi_{b} \vec{C} \xi_{b+1}^{-2}\right|=\bar{p}+2$. If $z_{1} y \in E$ for some $y \in V\left(\xi_{b} \vec{C} \xi_{b+1}^{-3}\right)$, then by Claim $2,\left|\xi_{b} \vec{C} y\right| \geq \bar{p}+2$, implying that $\left|I_{b}\right| \geq \bar{p}+5$, a contradiction. Hence, if $z_{1} y \in \Upsilon\left(I_{a}, I_{b}\right)$, then clearly $y=\xi_{b+1}^{-2}$. In particular, we have $z_{2}=\xi_{b+1}^{-2}$. Further, if $z_{1} y \in \Upsilon\left(I_{a}, I_{f}\right)$ for some $f \in\{1, \ldots, s\} \backslash\{b\}$, then by Lemma $2(\mathrm{a} 1),\left|I_{a}\right|+\left|I_{f}\right| \geq 2 \bar{p}+6$, 
that is $\left|I_{a}\right|+\left|I_{b}\right|+\left|I_{f}\right| \geq 3 \bar{p}+10$, contradicting Claim 1(a5). Thus, $z_{2}$ is a common vertex for all edges in $\Upsilon\left(I_{1}, \ldots, I_{s}\right)$. By Claim $3, G \backslash\left\{\xi_{1}, \ldots, \xi_{s}, z_{2}\right\}$ has at least $s+2$ components, contradicting $\tau \geq 1$.

Case a1.2. $\xi_{a}^{+} \xi_{b+1}^{-} \in E$.

By Claim 2, $\left|\xi_{a}^{+} \vec{C} \xi_{a+1}\right| \geq \bar{p}+2$ and $\left|\xi_{b} \vec{C} \xi_{b+1}^{-}\right| \geq \bar{p}+2$. If $\left|\xi_{a}^{+} \vec{C} \xi_{a+1}\right| \geq \bar{p}+3$ and $\left|\xi_{b} \vec{C} \xi_{b+1}^{-}\right| \geq \bar{p}+3$, then $\left|I_{a}\right|+\left|I_{b}\right| \geq 2 \bar{p}+8$, contradicting Claim 1(a1). Hence, we can assume w.l.o.g. that $\left|\xi_{b} \vec{C} \xi_{b+1}^{-}\right|=\bar{p}+2$, that is $\left|I_{b}\right|=\bar{p}+3$ and $\left|I_{a}\right| \geq \bar{p}+3$. Further, we have $\xi_{b}^{+} \xi_{a}, \xi_{b}^{+} \xi_{b+1} \notin E$ (by Claim 4 ) and $\xi_{b}^{+} \xi_{a}^{+} \notin E$ (by Claim 2).

Case a1.2.1. $N\left(\xi_{b}^{+}\right) \nsubseteq V(C)$.

Let $Q=\xi_{b}^{+} \vec{Q} v$ be a longest path in $G$ with $V(Q) \cap V(C)=\left\{\xi_{b}^{+}\right\}$. Since $C$ is extreme, we have $V(Q) \cap V(P)=\emptyset$. Next, since $P$ is a longest path in $G \backslash C$, we have $|Q| \leq \bar{p}+1$. Further, recalling that $\xi_{b}^{+} \xi_{a}, \xi_{b}^{+} \xi_{b+1}, \xi_{b}^{+} \xi_{a}^{+} \notin E$ (see Case a1.2), we conclude that $v \xi_{a}, v \xi_{b+1}, v \xi_{a}^{+} \notin E$, as well. If $v y \notin E$ for each $y \in\left(\xi_{b}^{+2} \vec{C} \xi_{b+1}^{-}\right)$, then clearly

$$
N(v) \subseteq\left(V(Q) \cup\left\{\xi_{1}, \ldots, \xi_{s}\right\}\right) \backslash\left\{\xi_{a}, \xi_{b+1} \xi_{a}^{+}\right\},
$$

that is $d(v) \leq|Q|+s-2 \leq \bar{p}+s-1=\delta-1$, a contradiction. Now let $v y \in E$ for some $y \in V\left(\xi_{b}^{+2} \vec{C} \xi_{b+1}^{-}\right)$. Assume that $y$ is chosen so as to minimize $\left|\xi_{b}^{+} \vec{C} y\right|$. Since $C$ is extreme, we have $\left|\xi_{b}^{+} \vec{C} y\right| \geq|Q|+1$. Further, since

$$
\left|N(v) \cap V\left(y \vec{C} \xi_{b+1}^{-}\right)\right| \geq \delta-(s-2)-|Q|,
$$

we have

$$
\begin{aligned}
& \left|\xi_{b}^{+} \vec{C} \xi_{b+1}^{-}\right| \geq|Q|+1+2(\delta-s+1-|Q|) \\
= & 2 \delta-|Q|-2 s+3 \geq 2 \delta-\bar{p}-2 s+2=\bar{p}+2 .
\end{aligned}
$$

But then $\left|I_{b}\right| \geq \bar{p}+4$, a contradiction.

Case a1.2.2. $N\left(\xi_{b}^{+}\right) \subseteq V(C)$.

Since $\mu(\Upsilon)=1$ and $\xi_{b}^{+} \xi_{a}^{+} \notin E$, we have

$$
N\left(\xi_{b}^{+}\right) \subseteq V\left(\xi_{b}^{+2} \vec{C} \xi_{b+1}^{-}\right) \cup\left\{\xi_{1}, \ldots, \xi_{s}\right\} \backslash\left\{\xi_{a}, \xi_{b+1}\right\} .
$$

If $\xi_{a} \neq \xi_{b+1}$, then $d\left(\xi_{b}^{+}\right) \leq \bar{p}+s-1=\delta-1$, a contradiction. Hence $\xi_{a}=\xi_{b+1}$.

Case a1.2.2.1. $\left|I_{f}\right|=\bar{p}+2$ for some $f \in\{1, \ldots, s\} \backslash\{a, b\}$.

If $N\left(\xi_{f}^{+}\right) \subseteq V(C)$, then as indicated above,

$$
d\left(\xi_{f}^{+}\right) \leq s-1+\left|\xi_{f}^{+} \vec{C} \xi_{f+1}^{-}\right|=\bar{p}+s-1=\delta-1,
$$

a contradiction. If $N\left(\xi_{f}^{+}\right) \nsubseteq V(C)$, then we can argue as in Case a1.2.1.

Case a1.2.2.2. $\left|I_{i}\right| \geq \bar{p}+3$ for each $i \in\{1, \ldots, s\} \backslash\{a, b\}$.

If $s \geq 4$, then

$$
|C|=\sum_{i=1}^{s}\left|I_{i}\right| \geq s(\bar{p}+3)=(\delta-\bar{p})(\bar{p}+3)
$$




$$
=2 \delta+2 \bar{p}+4+(\delta-\bar{p}-4)(\bar{p}+1) \geq 2 \delta+4,
$$

contradicting (1). Hence, $s \leq 3$. Moreover, if $s=3$, then by Claim 1(a5), $\left|I_{1}\right|=\left|I_{2}\right|=\left|I_{3}\right|=$ $\bar{p}+3$.

Case a2. $z_{1} z_{3}, z_{2} z_{3} \in \Upsilon\left(I_{1}, \ldots, I_{s}\right)$, where $z_{3} \in V\left(I_{f}^{*}\right)$ and $f \in\{1, \ldots, s\} \backslash\{a, b\}$.

Assume w.l.o.g. that $\xi_{a}, \xi_{b}, \xi_{f}$ occur on $\vec{C}$ in a consecutive order. Put

$$
\begin{gathered}
\left|\xi_{a} \vec{C} z_{1}\right|=d_{1},\left|z_{1} \vec{C} \xi_{a+1}\right|=d_{2},\left|\xi_{b} \vec{C} z_{2}\right|=d_{3}, \\
\left|z_{2} \vec{C} \xi_{b+1}\right|=d_{4},\left|\xi_{f} \vec{C} z_{3}\right|=d_{5},\left|z_{3} \vec{C} \xi_{f+1}\right|=d_{6} .
\end{gathered}
$$

By Claim 2,

$$
\begin{aligned}
& d_{1}+d_{3} \geq \bar{p}+3, d_{1}+d_{5} \geq \bar{p}+3, d_{2}+d_{4} \geq \bar{p}+3, \\
& d_{2}+d_{6} \geq \bar{p}+3, d_{3}+d_{5} \geq \bar{p}+3, d_{4}+d_{6} \geq \bar{p}+3 .
\end{aligned}
$$

Summing up, we get

$$
2 \sum_{i=1}^{6} d_{i}=2\left(\left|I_{a}\right|+\left|I_{b}\right|+\left|I_{f}\right|\right) \geq 6(\bar{p}+3) .
$$

On the other hand, by Claim 1(a5), $\left|I_{a}\right|+\left|I_{b}\right|+\left|I_{f}\right| \leq 3(\bar{p}+3)$, implying that $d_{1}=d_{2}=$ $\ldots=d_{6}=(\bar{p}+3) / 2$ and $\bar{p}$ is odd. Hence $d_{i} \geq 2$ and using Claim 3, we can state that $G \backslash\left\{\xi_{1}, \ldots, \xi_{s}, z_{1}, z_{2}\right\}$ has at least $s+3$ components, contradicting $\tau \geq 1$. Claim 6 is proved.

Claim 7. Either $\mu(\Upsilon)=1$ or $\mu(\Upsilon)=3$.

Proof. The proof is by contradiction. If $\mu(\Upsilon)=0$, then $G \backslash\left\{\xi_{1}, \ldots, \xi_{s}\right\}$ has at least $s+1$ components, contradicting $\tau \geq 1$. Let $\mu(\Upsilon) \geq 1$.

Case a1. $\mu=2$.

By Claim 5, $\Upsilon\left(I_{1}, \ldots, I_{s}\right)$ consists of two crossing intermediate independent edges $w_{1} w_{2} \in$ $\Upsilon\left(I_{f}, I_{g}\right)$ and $w_{3} w_{4} \in \Upsilon\left(I_{f}, I_{h}\right)$ for some distinct $f, g, h$. Assume that both $\xi_{f}, \xi_{g}, \xi_{h}$ and $w_{1}, w_{3}, w_{2}, w_{4}$ occur on $\vec{C}$ in a consecutive order. Put

$$
\begin{gathered}
Q=\xi_{f} \vec{C} w_{1} w_{2} \vec{C} w_{4} w_{3} \vec{C} \xi_{g} x_{2} \overleftarrow{P} x_{1} \xi_{h+1} \vec{C} \xi_{f}, \\
\left|\xi_{f} \vec{C} w_{1}\right|=d_{1},\left|w_{1} \vec{C} w_{3}\right|=d_{2},\left|w_{3} \vec{C} \xi_{f+1}\right|=d_{3}, \\
\left|\xi_{g} \vec{C} w_{2}\right|=d_{4},\left|w_{2} \vec{C} \xi_{g+1}\right|=d_{5},\left|\xi_{h} \vec{C} w_{4}\right|=d_{6},\left|w_{4} \vec{C} \xi_{h+1}\right|=d_{7}
\end{gathered}
$$

Clearly, $|Q|=|C|-d_{2}-d_{4}-d_{7}+\bar{p}+4$. Since $|Q| \leq|C|$, we have $d_{2}+d_{4}+d_{7} \geq \bar{p}+4$. If $d_{3}+d_{6} \geq \bar{p}+3$ and $d_{1}+d_{5} \geq \bar{p}+3$, then $\sum_{i=1}^{7} d_{i}=\left|I_{f}\right|+\left|I_{g}\right|+\left|I_{h}\right| \geq 3 \bar{p}+10$, contradicting Claim 1(a5). Otherwise, either $d_{3}+d_{6} \leq \bar{p}+2$ or $d_{1}+d_{5} \leq \bar{p}+2$, say $d_{3}+d_{6} \leq \bar{p}+2$. Further, if either $d_{7}=1$ or $\xi_{h+1}^{-} w_{3} \in E$, then by Claim $2, d_{3} \geq \bar{p}+2$, that is $d_{3}+d_{6} \geq \bar{p}+3$, a contradiction. Hence, $d_{7} \geq 2$ and $\xi_{h+1}^{-} w_{3} \notin E$. By Claim $4, \xi_{h+1}^{-} \xi_{f+1}, \xi_{h+1}^{-} \xi_{h} \notin E$. If $\left|I_{h}\right| \geq \bar{p}+4$, then taking into account that $\left|I_{f}\right|+\left|I_{g}\right| \geq 2 \bar{p}+6$ (by Claim 1(a1)), we get $\left|I_{f}\right|+\left|I_{g}\right|+\left|I_{h}\right| \geq 3 \bar{p}+10$, contradicting Claim 1(a5). Hence, $\left|I_{h}\right| \leq \bar{p}+3$. By a symmetric argument, $\left|I_{g}\right| \leq \bar{p}+3$.

Case a1.1. $N\left(\xi_{h+1}^{-}\right) \subseteq V(C)$. 
If $\xi_{h+1}^{-} w_{2} \notin E$, then recalling that $\mu(\Upsilon)=2$, we get

$$
N\left(\xi_{h+1}^{-}\right) \subseteq V\left(w_{4} \vec{C} \xi_{h+1}^{-2}\right) \cup\left\{\xi_{1}, \ldots, \xi_{s}\right\} \backslash\left\{\xi_{f+1}, \xi_{h}\right\},
$$

implying that $\left|N\left(\xi_{h+1}^{-}\right)\right| \leq \bar{p}+s-1=\delta-1$, a contradiction. Now let $\xi_{h+1}^{-} w_{2} \in E$. By Claim 1(a1 and a5), $\left|I_{f}\right|=\left|I_{g}\right|=\left|I_{h}\right|=\bar{p}+3$. Moreover, by Claim $2, d_{5}=\bar{p}+2$ and $d_{4}=1$. Then, for the same reason, $d_{1}=\bar{p}+2$, implying that $\left|I_{a}\right| \geq \bar{p}+4$, a contradiction.

Case a1.2. $N\left(\xi_{h+1}^{-}\right) \nsubseteq V(C)$.

We can argue as in the proof of Claim 6 (Case a1.2.1).

Case a2. $\mu(\Upsilon) \geq 4$.

By Claim 5, there are at least four pairwise crossing intermediate independent edges in $\Upsilon\left(I_{1}, \ldots, I_{s}\right)$, which is impossible. Claim 7 is proved.

Claim 8. If $\mu(\Upsilon)=1$, then either $n \equiv 1(\bmod 3)$ with $c \geq 2 \delta+2$ or $n \equiv 1(\bmod 4)$ with $c \geq 2 \delta+3$ or $n \equiv 2(\bmod 3)$ with $c \geq 2 \delta+3$.

Proof. By Claim 6, $s \leq 3$ and either $\xi_{a}^{+} \xi_{b+1}^{-} \in E$ or $\xi_{a+1}^{-} \xi_{b}^{+} \in E$, say $\xi_{a+1}^{-} \xi_{b}^{+} \in E$.

Case a1. $s=2$.

It follows that $\delta=\bar{p}+s=\bar{p}+2$. Let $a=1$ and $b=2$. By Claim 2, $\left|\xi_{1} \vec{C} \xi_{2}^{-}\right| \geq \bar{p}+2$ and $\left|\xi_{2}^{+} \vec{C} \xi_{1}\right| \geq \bar{p}+2$, implying that $\left|I_{i}\right| \geq \bar{p}+3(i=1,2)$.

Case a1.1. $\left|I_{1}\right|=\bar{p}+4$ and $\left|I_{2}\right|=\bar{p}+3$.

If $V(G)=V(C \cup P)$, then $n=3 \bar{p}+8=3 \delta+2 \equiv 2(\bmod 3)$ with $c=2 \bar{p}+7=2 \delta+3$, and we are done. Otherwise $N\left(v_{1}\right) \nsubseteq V(C \cup P)$ for some $v_{1} \in V(C \cup P)$. Observing that $x_{1} x_{2} \in E$ and recalling that $P$ is a longest path in $V(G \backslash C)$, we conclude that $v_{1} \notin V(P)$. Choose a longest path $Q=v_{1} \vec{Q} v_{2}$ with $V(Q) \cap V(C)=\left\{v_{1}\right\}$. Clearly, $1 \leq|Q| \leq \bar{p}+1=\delta-1$ and $N\left(v_{2}\right) \subseteq V(C \cup Q)$.

Case a1.1.1. $v_{1} \in V\left(\xi_{2}^{+2} \vec{C} \xi_{1}^{-}\right)$.

By Claim 1(a6), $N\left(v_{2}\right) \cap V\left(I_{1}^{*}\right)=\emptyset$, that is $N\left(v_{2}\right) \subseteq V\left(I_{1}\right) \cup V(Q)$. Assume that $v_{1}$ is chosen so as to minimize $\left|v_{1} \vec{C} \xi_{1}\right|$, implying that $N\left(v_{2}\right) \cap V\left(v_{1} \vec{C} \xi_{1}^{-}\right)=\emptyset$. Clearly, $\left|v_{1} \vec{C} \xi_{1}\right| \leq \bar{p}+1$. Then by Claim $4, v_{1} \xi_{2} \notin E$ and therefore, $v_{2} \xi_{2} \notin E$, as well.

Case a1.1.1.1. $v_{2} \xi_{1} \in E$.

It follows that $N\left(v_{2}\right) \subseteq V(Q) \cup V\left(\xi_{2}^{+} \vec{C} v_{1}^{-}\right) \cup\left\{\xi_{1}\right\}$. Since $C$ is extreme and $v_{2} \xi_{1} \in E$, we have $\left|v_{1} \vec{C} \xi_{1}\right| \geq|Q|+1$. If $N\left(v_{2}\right) \subseteq V(Q) \cup\left\{\xi_{1}\right\}$, then clearly $|Q| \geq \delta-1=\bar{p}+1$ and therefore, $\left|v_{1} \vec{C} \xi_{1}\right| \geq \bar{p}+2$. But then $\left|I_{2}\right| \geq \bar{p}+4$, a contradiction. Hence, $N\left(v_{2}\right) \nsubseteq V(Q) \cup\left\{\xi_{1}\right\}$, that is $v_{2} y \in E$ for some $y \in V\left(\xi_{2}^{+} \vec{C} v_{1}^{-}\right)$. Assume that $y$ is chosen so as to minimize $\left|y \vec{C} v_{1}\right|$. Observing that $\left|y \vec{C} v_{1}\right| \geq|Q|+1$ and $\delta=\left|\xi_{2}^{+} \vec{C} \xi_{1}\right| \geq 4$, we get

$$
\left|\xi_{2}^{+} \vec{C} \xi_{1}\right| \geq 2(|Q|+1)+2(\delta-|Q|-2)=2 \delta-2 \geq \delta+2=\bar{p}+4
$$

a contradiction.

Case a1.1.1.2. $v_{2} \xi_{1} \notin E$. 
It follows that $N\left(v_{2}\right) \subseteq V(Q) \cup V\left(\xi_{2}^{+} \vec{C} v_{1}^{-}\right)$. If $N\left(v_{2}\right) \subseteq V(Q)$, then $|Q| \geq \delta=\bar{p}+2$, a contradiction. Otherwise $v_{2} y \in E$ for some $y \in V\left(\xi_{2}^{+} \vec{C} v_{1}^{-}\right)$. Assume that $y$ is chosen so as to minimize $\left|y \vec{C} v_{1}\right|$. Since $\left|y \vec{C} v_{1}\right| \geq|Q|+1$, we have

$$
\left|\xi_{2}^{+} \vec{C} v_{1}\right| \geq|Q|+1+2(\delta-|Q|-1)=2 \delta-|Q|-1 \geq \delta=\bar{p}+2
$$

But then $\left|I_{b}\right| \geq 4$, a contradiction.

Case a1.1.2. $v_{1} \in V\left(\xi_{1}^{+} \vec{C} \xi_{2}^{-3}\right)$.

By Claim 1(a6), $N\left(v_{2}\right) \cap V\left(I_{2}^{*}\right)=\emptyset$, that is $N\left(v_{2}\right) \subseteq V(Q) \cup V\left(I_{1}\right)$. Assume that $v_{1}$ is chosen so as to minimize $\left|\xi_{1} \vec{C} v_{1}\right|$, implying that $N\left(v_{2}\right) \cap V\left(\xi_{1}^{+} \vec{C} v_{1}^{-}\right)=\emptyset$. Clearly, $\left|\xi_{1} \vec{C} v_{1}\right| \leq \bar{p}+1$. Then by Claim $4, v_{1} \xi_{2} \notin E$ and therefore, $v_{2} \xi_{2} \notin E$.

Case a1.1.2.1. $\xi_{2}^{+} \xi_{2}^{-2} \in E$.

By Claim $3, v_{1} \xi_{2}^{-} \notin E$, implying that $v_{2} \xi_{2}^{-} \notin E$.

Case a1.1.2.1.1. $v_{2} \xi_{1} \in E$.

It follows that $N\left(v_{2}\right) \subseteq V(Q) \cup V\left(v_{1} \vec{C} \xi_{2}^{-2}\right) \cup\left\{\xi_{1}\right\}$. Since $C$ is extreme and $v_{2} \xi_{1} \in E$, we have $\left|\xi_{1} \vec{C} v_{1}\right| \geq|Q|+1$. If $N\left(v_{2}\right) \subseteq V(Q) \cup\left\{\xi_{1}\right\}$, then $|Q| \geq \delta-1=\bar{p}+1$ and therefore, $\left|\xi_{1} \vec{C} v_{1}\right| \geq \bar{p}+2$. But then $\left|I_{1}\right| \geq \bar{p}+5$, a contradiction. Hence, $N\left(v_{2}\right) \nsubseteq V(Q) \cup\left\{\xi_{1}\right\}$, that is $v_{2} y \in E$ for some $y \in V\left(v_{1}^{+} \vec{C} \xi_{2}^{-2}\right)$. Assume that $y$ is chosen so as to minimize $\left|v_{1} \vec{C} y\right|$. Observing that $\left|v_{1} \vec{C} y\right| \geq|Q|+1$ and $\delta=\left|\xi_{1} \vec{C} \xi_{2}^{-2}\right| \geq 4$, we get

$$
\left|\xi_{1} \vec{C} \xi_{2}^{-2}\right| \geq 2(|Q|+1)+2(\delta-|Q|-2)=2 \delta-2 \geq \delta+2=\bar{p}+4,
$$

a contradiction.

Case a1.1.2.1.2. $v_{2} \xi_{1} \notin E$.

It follows that $N\left(v_{2}\right) \subseteq V(Q) \cup V\left(v_{1} \vec{C} \xi_{2}^{-2}\right)$. If $N\left(v_{2}\right) \subseteq V(Q)$, then $|Q| \geq \delta=\bar{p}+2$, a contradiction. Otherwise $v_{2} y \in E$ for some $y \in V\left(v_{1}^{+} \vec{C} \xi_{2}^{-2}\right)$. By choosing $y$ so as to minimize $\left|v_{1} \vec{C} y\right|$, we get

$$
\left|v_{1} \vec{C} \xi_{2}^{-2}\right| \geq|Q|+1+2(\delta-|Q|-1)=2 \delta-|Q|-1 \geq \delta=\bar{p}+2
$$

This yields $\left|I_{a}\right| \geq \bar{p}+5$, a contradiction.

Case a1.1.2.2. $\xi_{2}^{+} \xi_{2}^{-2} \notin E$.

If $v_{2} \xi_{1} \in E$, then as in Case a1.1.2.1.1, $\left|\xi_{1} \vec{C} \xi_{2}^{-}\right| \geq \bar{p}+4$, contradicting the fact that $\left|I_{1}\right|=$ $\bar{p}+4$. Otherwise, as in Case a1.1.2.1.2, $\left|v_{1} \vec{C} \xi_{2}^{-}\right| \geq \bar{p}+2$. Since $\left|I_{1}\right|=\bar{p}+4$, we have $v_{1}=\xi_{1}^{+}$, $|Q|=\delta-1=\bar{p}+1$ and $v_{3}=\xi_{2}^{-}$. Moreover, we have $N\left(v_{2}\right)=\left(V(Q) \cup\left\{\xi_{2}^{-}\right\}\right) \backslash\left\{v_{2}\right\}$. Further, let $v$ be an arbitrary vertex in $V(Q) \backslash\left\{v_{1}\right\}$. Put $Q^{\prime}=v_{1} \vec{Q} v^{-} v_{2} \overleftarrow{Q} v$. Since $Q^{\prime}$ is another longest path with $V\left(Q^{\prime}\right) \cap V(C)=\left\{v_{1}\right\}$, we can suppose that $N(v)=\left(V(Q) \cup\left\{\xi_{2}^{-}\right\}\right) \backslash\{v\}$ for each $v \in V(Q) \backslash\left\{v_{1}\right\}$. Furthermore, if $\xi_{1} y \in E$ for some $y \in V\left(\xi_{1}^{+2} \vec{C} \xi_{2}^{-2}\right)$, then

$$
\xi_{1} x_{1} \vec{P} x_{2} \xi_{2} \xi_{2}^{+} \xi_{2}^{-} v_{2} \overleftarrow{Q} v_{1} \vec{C} y \xi_{1}
$$


is longer than $C$, a contradiction. Hence, $\xi_{1} y \notin E$ for each $y \in V\left(\xi_{1}^{+2} \vec{C} \xi_{2}^{-2}\right)$. Analogously, if $y \xi_{2} \in E$ for some $y \in V\left(\xi_{1}^{+} \vec{C} \xi_{2}^{-2}\right)$, then

$$
\xi_{1} x_{1} \vec{P} x_{2} \xi_{2} y \overleftarrow{C} \xi_{1}^{+} \vec{Q} v_{2} \xi_{2}^{-} \xi_{2}^{+} \vec{C} \xi_{1}
$$

is longer than $C$, a contradiction. Hence, $y \xi_{2} \notin E$ for each $y \in V\left(\xi_{1}^{+} \vec{C} \xi_{2}^{-2}\right)$. But then $G \backslash\left\{\xi_{1}^{+}, \xi_{2}^{-}\right\}$has at least three components, contradicting $\tau \geq 1$.

Case a1.1.3. $v_{1}=\xi_{2}^{-2}$.

By Claim $1(\mathrm{a} 6), N\left(v_{2}\right) \subseteq V\left(I_{1}\right)$. If $v_{2} y \in E$ for some $y \in V\left(\xi_{1}^{+} \vec{C} v_{1}^{-}\right)$, then we can argue as in Case a1.1.2. Hence, $N\left(v_{2}\right) \subseteq V(Q) \cup\left\{\xi_{1}, \xi_{2}\right\}$. If $v_{2} \xi_{2} \in E$, then

$$
\xi_{1} x_{1} \vec{P} x_{2} \xi_{2} v_{2} \overleftarrow{Q} v_{1} \xi_{2}^{-} \xi_{2}^{+} \vec{C} \xi_{1}
$$

is longer than $C$, a contradiction. Then clearly, $v_{2} \xi_{1} \in E$ and $N\left(v_{2}\right) \subseteq V(Q) \cup\left\{\xi_{1}\right\}$. Furthermore, we have $|Q| \geq \delta-1$, implying that $\left|\xi_{1} \vec{C} v_{1}\right| \geq|Q|+1 \geq \delta$. Since $\left|\xi_{1} \vec{C} v_{1}\right|=\delta$, we have $|Q|=\delta-1=\bar{p}+1$ and $N\left(v_{2}\right)=\left(V(Q) \cup\left\{\xi_{1}\right\}\right) \backslash\left\{v_{2}\right\}$. Moreover, as in Case 1.1.2.2, we have $N(v)=\left(V(Q) \cup\left\{\xi_{1}\right\}\right) \backslash\{v\}$ for each $v \in V(Q) \backslash\left\{v_{1}\right\}$. Now consider an arbitrary vertex $y \in V\left(\xi_{1}^{+} \vec{C} \xi_{2}^{-3}\right)$. Clearly, $\left|\xi_{1} \vec{C} y\right| \leq \bar{p}+1$. By Claim 2, y $\xi_{2}^{+} \notin E$. Next, by Claim 4 , $y \xi_{2} \notin E$. Further, if $y \xi_{2}^{-} \in E$, then

$$
\xi_{1} x_{1} \vec{P} \xi_{2} \xi_{2} \xi_{2}^{+} \xi_{2}^{-} y \vec{C} \xi_{2}^{-2} \vec{Q} v_{2} \xi_{1}
$$

is longer than $C$, a contradiction. Finally, since $\mu(\Upsilon)=1$, we have $y v \notin E$ for each $v \in V\left(\xi_{2}^{+2} \vec{C} \xi_{1}^{-}\right)$. But then $G \backslash\left\{\xi_{1}, \xi_{2}^{-2}\right\}$ has at least three components, contradicting $\tau \geq 1$.

Case a1.1.4. $v_{1}=\xi_{1}$.

If $v_{2} v_{3} \in E$ for some $v_{3} \in V\left(\xi_{2}^{+2} \vec{C} \xi_{1}^{-}\right) \cup V\left(\xi_{1}^{+} \vec{C} \xi_{2}^{-2}\right)$, then we can argue as in Cases a1.1.1-a1.1.3. Otherwise $v_{2} v_{3} \in E$ for some $v_{3} \in\left\{\xi_{2}^{-}, \xi_{2}^{+}, \xi_{2}\right\}$. If $v_{3} \in\left\{\xi_{2}, \xi_{2}^{+}\right\}$, then we can show, as in Case a1.1.3, that $G \backslash\left\{\xi_{1}, v_{3}\right\}$ has at least three components, contradicting $\tau \geq 1$. Now let $v_{3}=\xi_{2}^{-}$. Consider an arbitrary vertex $v \in V(Q) \backslash\left\{v_{1}\right\}$. Since $C$ is extreme, we have $N(v) \cap\left\{\xi_{2}, \xi_{2}^{+}\right\}=\emptyset$. Next, if $v y \in E$ for some $y \in V(C) \backslash\left\{\xi_{1}, \xi_{2}, \xi_{2}^{-}, \xi_{2}^{+}\right\}$, then we can argue as in Cases a1.1.1-a1.1.3. Thus, we can assume that $N(v) \subseteq V(Q) \cup\left\{\xi_{2}^{-}\right\}$, implying that $|Q| \geq \delta-1=\bar{p}+1$. Let $w \in V\left(\xi_{1}^{+} \vec{C} \xi_{2}^{-3}\right)$. Since $\left|\xi_{1} \vec{C} w\right| \leq \bar{p}+1$, we have $w \xi_{2}^{+} \notin E$ (by Claim 2) and $w \xi_{2} \notin E$ (by Claim 4). Recalling also that $\mu(\Upsilon)=1$, we conclude that $N(v) \subseteq V\left(\xi_{1} \vec{C} \xi_{2}^{-}\right)$. If $\xi_{2}^{-2} \xi_{2}, \xi_{2}^{-2} \xi_{2}^{+} \notin E$, then clearly $G \backslash\left\{\xi_{1}, \xi_{2}^{-}\right\}$has at least three components, contradicting $\tau \geq 1$. Hence, either $\xi_{2}^{-2} \xi_{2} \in E$ or $\xi_{2}^{-2} \xi_{2}^{+} \in E$.

Case a1.1.4.1. $\xi_{2}^{-2} \xi_{2} \in E$.

If $\xi_{2}^{-2} \xi_{2}^{+} \notin E$, then $G \backslash\left\{\xi_{1}, \xi_{2}, \xi_{2}^{-}\right\}$has at least four components, contradicting $\tau \geq 1$. Hence, $\xi_{2}^{-2} \xi_{2}^{+} \in E$, that is $\left\langle\xi_{2}, \xi_{2}^{-}, \xi_{2}^{-2}, \xi_{2}^{+}\right\rangle$is a complete graph. If $V(G)=V(C \cup P \cup Q)$, then $n=4 \delta+1 \equiv 1(\bmod 4)$ with $c=2 \delta+3$, and we are done. Otherwise, as in previous cases, we can show that $\tau<1$, a contradiction.

Case a1.1.4.2. $\xi_{2}^{-2} \xi_{2}^{+} \in E$.

If $\xi_{2}^{-2} \xi_{2} \notin E$, then $G \backslash\left\{\xi_{1}, \xi_{2}^{-}, \xi_{2}^{+}\right\}$has at least four components, contradicting $\tau \geq 1$. Otherwise $\left\langle\xi_{2}, \xi_{2}^{-}, \xi_{2}^{-2}, \xi_{2}^{+}\right\rangle$is a complete graph and we can argue as in Case a1.1.4.1. 
Case a1.1.5. $v_{1} \in\left\{\xi_{2}, \xi_{2}^{-}, \xi_{2}^{+}\right\}$.

Since $C$ is extreme, we have $v_{2} \notin\left\{\xi_{2}, \xi_{2}^{-}, \xi_{2}^{+}\right\}$and therefore, we can argue as in Cases a1.1.1-1.1.4.

Case a1.2. $\left|I_{1}\right|=\left|I_{2}\right|=\bar{p}+3$.

We can show that $n=3 \delta+1 \equiv 1(\bmod 3)$ with $c=2 \delta+2$, by arguing as in Case a1.1.

Case a2. $s=3$.

By Claim 6, $\left|I_{1}\right|=\left|I_{2}\right|=\left|I_{3}\right|=\bar{p}+3=\delta$ and $\xi_{2}^{-} \xi_{2}^{+} \in E$. If $\delta \geq 4$, then $c=3 \delta \geq 2 \delta+4$, contradicting (1). Hence $\delta=3$ and therefore, $\bar{p}=0$. Put

$$
C=\xi_{1} w_{1} w_{2} \xi_{2} w_{3} w_{4} \xi_{3} w_{5} w_{6} \xi_{1}
$$

where $w_{2} w_{3} \in E$. Using Claims 2-5, we can show that

$$
N_{C}\left(w_{1}\right)=\left\{w_{2}, \xi_{1}, \xi_{3}\right\}, N_{C}\left(w_{6}\right)=\left\{w_{5}, \xi_{1}, \xi_{3}\right\} .
$$

Analogous relations hold for $w_{4}, w_{5}$. If $V(G \backslash C)=\left\{x_{1}\right\}$, then $n=10 \equiv 1(\bmod 3)$ with $c=9=2 \delta+3>2 \delta+2$, and we are done. Otherwise $N(y)=\left\{v_{1}, v_{2}, v_{3}\right\}$ for some $y \in V(G \backslash C) \backslash\left\{x_{1}\right\}$ with $N(y) \subseteq V(C)$. Since $C$ is extreme, it is not hard to see that either $N(y)=\left\{w_{2}, \xi_{1}, \xi_{3}\right\}$ or $N(y)=\left\{w_{3}, \xi_{1}, \xi_{3}\right\}$ or $N(y)=\left\{\xi_{1}, \xi_{2}, \xi_{3}\right\}$. But then $G \backslash N(y)$ has at least four components, contradicting $\tau \geq 1$. Claim 8 is proved.

Claim 9. If $\mu=3$, then $G$ is the Petersen graph, that is $n=10 \equiv 1(\bmod 3)$ with $c \geq 2 \delta+2$.

Proof. By Claim 5, $\Upsilon\left(I_{1}, \ldots, I_{s}\right)$ contains three pairwise crossing intermediate independent edges $e_{1}, e_{2}, e_{3}$. Let $e_{1}=w_{1} w_{2}, e_{2}=w_{3} w_{4}$ and $e_{3}=w_{5} w_{6}$. If $w_{1}, w_{3}, w_{5} \in V\left(I_{f}^{*}\right)$ for some $f \in\{1, \ldots, s\}$, then we can argue as in proof of Claim 7. Otherwise we can assume w.l.o.g. that $w_{1}, w_{3} \in V\left(I_{f}^{*}\right), w_{2}, w_{5} \in V\left(I_{g}^{*}\right)$ and $w_{4}, w_{6} \in V\left(I_{h}^{*}\right)$ for some distinct $f, g, h \in\{1, \ldots, s\}$, where both $\xi_{f}, \xi_{g}, \xi_{h}$ and $w_{1}, w_{3}, w_{5}, w_{2}, w_{4}, w_{6}$ occur on $\vec{C}$ in a consecutive order. By Claim 1(a1 and a5), $\left|I_{f}\right|=\left|I_{g}\right|=\left|I_{h}\right|=\bar{p}+3$ and $\left|I_{i}\right|=\bar{p}+2$ for each $i \in\{1, \ldots, s\} \backslash\{f, g, h\}$. Put

$$
\begin{aligned}
& \left|\xi_{f} \vec{C} w_{1}\right|=d_{1},\left|w_{1} \vec{C} w_{3}\right|=d_{2},\left|w_{3} \vec{C} \xi_{f+1}\right|=d_{3}, \\
& \left|\xi_{g} \vec{C} w_{5}\right|=d_{4},\left|w_{5} \vec{C} w_{2}\right|=d_{5},\left|w_{2} \vec{C} \xi_{g+1}\right|=d_{6}, \\
& \left|\xi_{h} \vec{C} w_{4}\right|=d_{7},\left|w_{4} \vec{C} w_{6}\right|=d_{8},\left|w_{6} \vec{C} \xi_{h+1}\right|=d_{9} .
\end{aligned}
$$

If $d_{3}+d_{7} \geq \bar{p}+3, d_{1}+d_{6} \geq \bar{p}+3$ and $d_{4}+d_{9} \geq \bar{p}+3$, then clearly $\left|I_{f}\right|+\left|I_{g}\right|+\left|I_{h}\right| \geq 3 \bar{p}+12$, a contradiction. Otherwise we can assume w.l.o.g. that $d_{3}+d_{7} \leq \bar{p}+2$. Further, if either $d_{1} \geq 2$ or $d_{9} \geq 2$, then we can argue as in the proof of Claim 7 (Case a1.1). Hence, we can assume that $d_{1}=d_{9}=1$. By Claim $2, d_{4}=d_{6}=1$. For the same reason, using the fact that $d_{1}=d_{6}=1$, we get $d_{3}=d_{7}=1$.

Case a1. Either $\xi_{h+1} \neq \xi_{f}$ or $\xi_{f+1} \neq \xi_{g}$ or $\xi_{g+1} \neq \xi_{h}$.

Assume w.l.o.g. that $\xi_{h+1} \neq \xi_{f}$, implying that $\left|I_{f-1}\right|=\bar{p}+2$. By Claim $5, \xi_{f}^{-} y \notin E$ for each $y \in V\left(I_{i}^{*}\right)$ and $i \in\{1, \ldots, s\} \backslash\{f-1\}$. Moreover, by Claim $4, \xi_{f}^{-} y \notin E$ for each $y \in\left\{\xi_{f+1}, \xi_{h}\right\}$. If $N\left(\xi_{f}^{-}\right) \subseteq V(C)$, then $d\left(\xi_{f}^{-}\right) \leq \delta-1$, a contradiction. Otherwise we can 
argue as in the proof of Claim 6 (Case a1.2.1).

Case a2. $\xi_{h+1}=\xi_{f}, \xi_{f+1}=\xi_{g}, \xi_{g+1}=\xi_{h}$.

It follows that $s=3$. Assume w.l.o.g. that $f=1, g=2$ and $h=3$.

Case a2.1. Either $d_{2} \geq 2$ or $d_{5} \geq 2$ or $d_{8} \geq 2$.

Assume w.l.o.g. that $d_{2} \geq 2$, that is $w_{1}^{+} \neq w_{3}$. If $\bar{p}=0$, then $\left|I_{1}\right|=3$, implying that $d_{2}=1$, a contradiction. Let $\bar{p} \geq 1$. By Claim $4, w_{1}^{+} \xi_{2}, w_{1}^{+} \xi_{3} \notin E$. If $N\left(w_{1}^{+}\right) \subseteq V(C)$, then by Claim $4, N\left(w_{1}^{+}\right) \subseteq V\left(w_{1}^{+2} \vec{C} w_{3}\right) \cup\left\{\xi_{1}\right\}$. Since $\left|I_{1}\right|=\bar{p}+3$, we have $\left|w_{1}^{+} \vec{C} w_{3}\right| \leq \bar{p}$. But then $d\left(w_{1}^{+}\right) \leq \bar{p}+1=\delta-2$, a contradiction. If $N\left(w_{1}^{+}\right) \nsubseteq V(C)$, then we can argue as in the proof of Claim 6 (Case a1.2.1).

Case a2.2. $d_{2}=d_{5}=d_{8}=1$.

It follows that $\left|I_{i}\right|=3(i=1,2,3)$, that is $\bar{p}=0, \delta=3$ and $c=9$. Clearly $\left\langle V(C) \cup\left\{x_{1}\right\}\right\rangle$ is the Petersen graph. If $V(G \backslash C) \neq\left\{x_{1}\right\}$, then it is not hard to see that $c \geq 10$, a contradiction. Otherwise, $n=10 \equiv 1(\bmod 3)$ with $c=9=2 \delta+3>2 \delta+2$. Claim 9 is proved.

Thus, the result holds from Claims 7,8,9.

Case 2. $\bar{p}=\delta-1$.

Clearly, $\left|N_{C}\left(x_{i}\right)\right| \geq 1(i=1,2)$.

Case 2.1. $x_{1} y_{1}, x_{2} y_{2} \in E$ for some distinct $y_{1}, y_{2} \in V(C)$.

We distinguish three main subcases.

Case 2.1.1. There exists a path $Q=z \vec{Q} y$ with $z \in V(P), y \in V(C) \backslash\left\{y_{1}, y_{2}\right\}$ and $V(Q) \cap V(C \cup P)=\{z, y\}$.

Assume w.l.o.g. that $y \in V\left(y_{1}^{+} \vec{C} y_{2}^{-}\right)$. Since $C$ is extreme, we have

$$
\left|y_{1} \vec{C} y\right| \geq\left|x_{1} \vec{P} z\right|+2,\left|y \vec{C} y_{2}\right| \geq\left|z \vec{P} x_{2}\right|+2,\left|y_{2} \vec{C} y_{1}\right| \geq \delta+1
$$

Summing up, we get $|C| \geq 2 \delta+4$, contradicting (1).

Case 2.1.2. There exists a path $Q=z \vec{Q} y$ with $z \in V\left(y_{1}^{+} \vec{C} y_{2}^{-}\right), y \in V\left(y_{2}^{+} \vec{C} y_{1}^{-}\right)$and $V(Q) \cap V(C \cup P)=\{z, y\}$.

By Claim 1(a1), $|C| \geq 2 \bar{p}+6=2 \delta+4$, contradicting (1).

Case 2.1.3. $G \backslash\left\{y_{1}, y_{2}\right\}$ has at least three components.

It follows that $\tau<1$, contradicting the hypothesis.

Case 2.2. $N_{C}\left(x_{1}\right)=N_{C}\left(x_{2}\right)=\{y\}$ for some $y \in V(C)$.

It follows that

$$
N\left(x_{1}\right)=(V(P) \cup\{y\}) \backslash\left\{x_{1}\right\}, N\left(x_{2}\right)=(V(P) \cup\{y\}) \backslash\left\{x_{2}\right\} .
$$

Moreover, $x_{1} \vec{P} v^{-} x_{2} \overleftarrow{P} v$ is a longest path in $G \backslash C$ for each $v \in V\left(x_{1}^{+} \vec{P} x_{2}\right)$. Since $G$ is 2connected, we have $w z \in E$ for some $w \in V(P)$ and $z \in V(C) \backslash\{y\}$. If $w=x_{1}$, then using the 
path $z x_{1} \vec{P} x_{2} y$, we can argue as in Case 2.1. Otherwise we can use the path $y x_{1} \vec{P} w^{-} x_{2} \overleftarrow{P} w z$

Case 3. $\bar{p} \geq \delta$.

Case 3.1. $x_{1} y_{1}, x_{2} y_{2} \in E$ for some distinct $y_{1}, y_{2} \in V(C)$.

Clearly, $\left|y_{1} \vec{C} y_{2}\right| \geq \delta+2$ and $\left|y_{2} \vec{C} y_{1}\right| \geq \delta+2$, which yields $|C| \geq 2 \delta+4$, contradicting (1).

Case 3.2. $N_{C}\left(x_{1}\right)=N_{C}\left(x_{2}\right)=\{y\}$ for some $y \in V(C)$.

Let $y_{1}, y_{2}, \ldots, y_{t}$ be the elements of $N_{P}^{+}\left(x_{2}\right)$ occurring on $\vec{P}$ in a consecutive order. Put $H=\left\langle V\left(y_{1}^{-} \vec{P} x_{2}\right)\right\rangle$ and

$$
P_{i}=x_{1} \vec{P} y_{i}^{-} x_{2} \overleftarrow{P} y_{i}(i=1, \ldots, t)
$$

Since $P_{i}$ is a longest path in $G \backslash C$ for each $i \in\{1, \ldots, t\}$, we can assume w.l.o.g. that $P$ is chosen so as to maximize $|V(H)|$. If $y_{i} z \in E$ for some $i \in\{1, \ldots, t\}$ and $z \in V(C) \backslash\{y\}$, then we can argue as in Case 3.1. Otherwise $N\left(y_{i}\right) \subseteq V(H) \cup\{y\}(i=1, \ldots, t)$, that is $\left|N_{H}\left(y_{i}\right)\right| \geq \delta-1(i=1, \ldots, t)$. By Lemma 3, for each distinct $u, v \in V(H)$, there is a path in $H$ of length at least $\delta-1$, connecting $u$ and $v$. Since $G$ is 2-connected, $H$ and $C$ are connected by two vertex disjoint paths. This means that there is a path $Q=y_{1} \vec{Q} y_{2}$ of length at least $\delta+1$ with $V(Q) \cap V(C)=\left\{y_{1}, y_{2}\right\}$. Further, we can argue as in Case 2.1.

Case 3.3. Either $N_{C}\left(x_{1}\right)=\emptyset$ or $N_{C}\left(x_{2}\right)=\emptyset$.

Assume w.l.o.g. that $N_{C}\left(x_{1}\right)=\emptyset$. By arguing as in Case 3.2, we can find a path $Q=y_{1} \vec{Q} y_{2}$ of length at least $\delta+2$ with $V(Q) \cap V(C)=\left\{y_{1}, y_{2}\right\}$, and the result follows immediately. Theorem 1 is proved.

\section{References}

[1] D. Bauer and E. Schmeichel, Long cycles in tough graphs, Technical Report 8612, Stevens Institute of Technology, 1986.

[2] J.A. Bondy and U.S.R. Murty, Graph Theory with Applications, Macmillan, London and Elsevier, New York 1976.

[3] V. Chvátal, "Tough graphs and hamiltonian circuits, Discrete Math., vol. 5, pp. 215-228 1973.

[4] G. A. Dirac, "Some theorems on abstract graphs", Proc. London, Math. Soc., vol. 2, pp. 69-81, 1952.

[5] H.-J. Voss, "Bridges of longest circuits and of longest paths in graphs", Beitrage zur Graphentheorie und deren Anwendungen, Vorgetr. auf dem. int. Kolloq., Oberhof (DDR), pp. 275-286, 1977.

Submitted 25.07.2018, accepted 20.11.2018. 


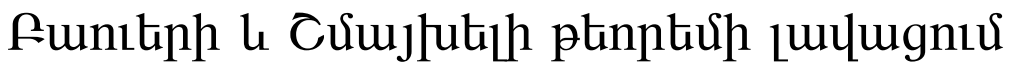

\author{
๙. Uhlnnnujua
}

\section{Uরuนhnนnıu}

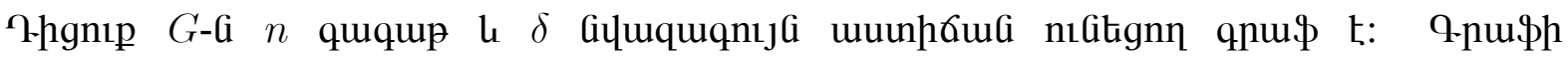

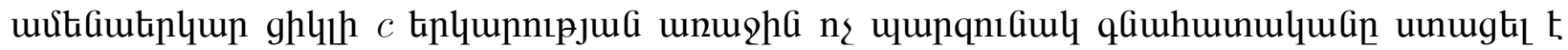

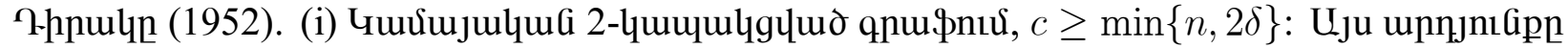

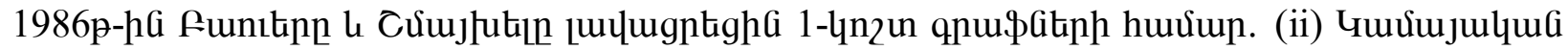

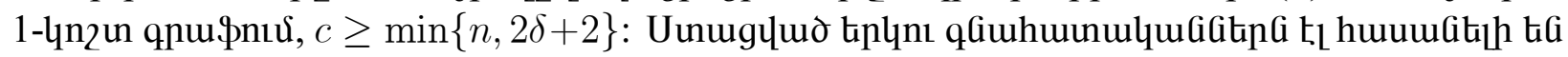

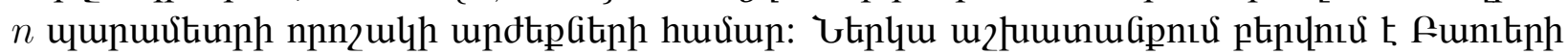

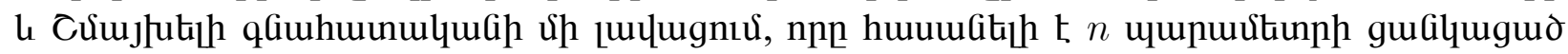
undtiph ntiupnus:

\section{Улучшение Теоремы Бауера и Шмейхеля}

\author{
Ж. Никогосяан
}

\begin{abstract}
Аннотация
Пусть $G$ является $n$ вершинным графром с минимальной степенью $\delta$. В 1952г. Аирак получил первую нетривиальную оценку Аля длины с Алиннейшего цикла графра $G$ : (i) В любом 2-связном графе, $c \geq \min \{n, 2 \delta\}$. Эту оценку в 1986г. Бауер и Шмейхель улучшили для 1-жестких графов: (ii) В любом 1-жестком графе, $c \geq \min \{n, 2 \delta+2\}$. Полученные оценки достигаемы для определенных значений параметра $n$. В настоящей работе предлагается улучшение оценки Бауера и Шмейхелья, которое неулучшаема для всех значений параметра $n$.
\end{abstract}

